The further history of the case showed lessening of the conjunctival congestion with the use of full correction in her glasses, but phlyctens used to appear till the menopause set in two years later.

There was no evident tonsillar trouble, nor any enlargement of cervical glands. Whether the disturbance of the system during the periods, in the presence of some source of infection, might be the cause of the appearance of phlyctens is a matter of opinion, but the fact that they used to disappear with the cessation of flow, and completely disappeared later on with the early onset of menopause, is a singular phenomenon to note.

One might mention here that on a few occasions congestion of the eyes was very slight. It never struck her to look for phlyctens on such occasions in a looking glass. Whether the scanty flow synchronized with that could not be accurately elucidated.

\title{
THE CIRCULATION OF THE INTRA-OCULAR FLUIDS
}

BY

\section{W. STEWART DUKe-Elder}

LONDON

IN his article "On the movement of the intraocular fluid as taught by Theodor Leber" (Brit. Jl. of Ophthal., Vol. XI, p. 236, 192 i), Priestley Smith, by agreeing with the essential principle of $\mathrm{my}$ views on the circulation of the intra-ocular fluids, has added the weight of his experience as a clinician to my comparative inexperience. Of the Continental writers who have concluded that the aqueous humour is a dialysate, Magitot has definitely committed himself to the assertion that this fluid is stagnant; Hamburger considers that no circulation through the pupil exists, but that a process of cellular interchange ("cellulärer Stoffwechsel") takes place throughout the vascularized tissues of the eye associated predominantly with the iris; and Weiss considers that the question is unproven, but leads one to infer that the existence of a circulation is very questionable. On the other hand, while holding that the aqueous humour is a dialysate, I maintain that a circulation does exist (see pages 9, 77, 89, 106, 108, etc.*), and Priestley Smith agrees with me.

It is only with regard to the cause of the circulation that we differ. Priestley Smith considers that the circulation is a

*?The page-references throughout refer to pages in my Monograph: "On the Nature of the Intra-ocular Fluids," 1927. 
"primary" one, determined by differences of pressure between the ciliary capillaries and the aqueous humour on the one hand, and the aqueous and the blood in the veins associated with the canal of Schlemm on the other; while I suggest that there is a balancing hydrostatic and osmotic pressure-equilibrium on either side of the capillary walls, which, as far as movement in mass is concerned, renders the aqueous humour "primarily stagnant," but which is continually being upset by changes in pressure occasioned by muscular action, the pulse-beat, etc. While there is thus at all times a free passage of diffusible substances through the capillary walls with a readjustment depending on colloidal impermeabilities, there is superimposed upon the dialysate a secondary or (so to say) " "pressure circulation," rendered possible by the delicately balanced pressure-equilibrium at the canal of Schlemm (the "safety-valve action" : see Brit. Jl. of Ophthal., Vol. X, p. 550,1926$)$. It is to be remembered that to all intents and purposes such a circulation will be continuous, although in actual fact the forces causing it act intermittently, and these will be quite capable of determining a flow of fluid so small as exists in the eye, a point upon whicn Priestley Smith rightly insists.

The same state of affairs exists throughout the body generally, and it seems to me, if we take into consideration the increased impermeability of the walls of the capillaries of the eye to colloidal micelles, that the aqueous humour bears a strictly parallel analogy to the tissue fluids (see p. 108 : "As with their formation, so with their metaboiism and circulation, the intraocular fluids and the tissue fluids of the body are regulated by the same factors'). The tissue fluids are formed by the balanced inter-action of osmotic and hydrostatic forces (Starling), and they depend for their "circulation" upon muscular contraction, changes in abdominal and thoracic pressure, the movements of organs during activity, and other secondary factors, without the intervention of which they stagnate, although at the same time metabolic interchange proceeds unaffected. It is well known that if muscular movement be abolished, oedema results readily in the dependent parts, as the leg (see most recently, Carriere and Rehberg, Skand. Arch. f. Physiol., Vol. XLIV, p. 20, 1923); and this seems to me to provide an analogy with the rise in the intra-ocular pressure which occurs in chronic glaucoma during the night especially towards morning, when movement of both the extra-ocular and intra-ocular muscles, which during the waking hours has been practically continuous, has been abolished for some time.

Such was the general conception of my thesis, and it seems unnecessary to amplify it further. In the matter of accounting for, and insisting upon a circulation combined with the conception of dialysis, it is new, and has not (to my knowledge) been offered before. Priestley Smith considers that I have "not made my position quite definite," and suggests that at one moment 'I deny the existence of a circulation 
and at the next "make considerable concessions to old beliefs." I would dissociate myself from an attempt to run with the hare and hunt with the hounds, and can only apologize for my lack of clarity of expression, but at the same time would point out that in my monograph the necessity of postulating a circulation was insisted upon in page 9 as well as page 110. I will repeat and amplify the passage which was quoted: $I$, therefore, concelive of the eye as containing a fluid in membrane-equilibrium with the capillary blood. As such it must be essentially stagnant. (So far I agree with Magitot, Hamburger, and the implications of Weiss.) At the same time (p. 106) the evidence, physiological and pathological (which Priestley Smith quotes, and the greater part of which is essentially the same as I myself brought forward), seems to demonstrate that the existence of a circulation cannot be denied (so far I agree with the conceptions of Leber, p. 108). . . . The truth is to be found in a reconciliation and unification of the two schools of thought (p. 108).

Priestley Smith suggests that "the blood pressure is higher in the ciliary arteries than in Schlemm's canal and in the iris veins, while the aqueous pressure hardly differs at all in the two regions : it is difficult, therefore, to see how osmotic equilibrium can exist in the two regions at the same time." I know of no instance in the body where thermo-dynamical equilibrium exists through the walls of veins; it is through the walls of capillaries that metabolic changes take place. Chemical analyses, if they have demonstrated anything, have shown that the aqueous humour is in thermodynamical equilibrium with the capillary blood (for example, sugar, p. 28: "the concentration appears to be related more closely to the arterial than to the venous plasma"; salt, p. 30 : "the aqueous comes into relation with the capillary plasma .... the capillary plasma is in this respect more nearly related to the arterial than the venous condition"; osmotic pressure, p. 56 : "the osmotic concentration . . . . appears to lie between the point of equilibrium with arterial and that with venous blood, being much more closely related to the arterial"'; and so on). There is no evidence of equilibrium with venous blood or ever any question of its occurring.

There seems little necessity to examine Priestley Smith's arguments individually, since his main contention, the existence of a circulation, is admitted, and since nowhere does he bring forward any evidence that the aqueous humour is not a product of a thermodynamical equilibrium, or that the circulation which is present in the eye is not determined secondarily by factors quite independent of those governing its formation. There are one or two points, however, which he brings up which may bear looking into.

I agree with Priestley Smith that Seidel's experimental work has to a large extent refuted the arguments whereupon Hamburger based his conception of a "physiological barrier" existing at the pupil; but Seidel's measurements of the venous pressure in the episcleral veins, which purported to demonstrate a pressuregradient existing under normal conditions between the aqueous humour and the venous blood, are decidedly open to question. The measurements were taken of veins outside the sclerotic, and had no relation with the pressure-equilibrium in the eye. Seidel's measurements were approximately correct: the blood in these veins outside the eye is at lower pressure than the chamber-pressure, but the blood in the veins inside the eye and through the thickness of the sclerotic is in normal 
circumstances at a higher pressure (see Brit. Jl. of Ophthal., Vol. X, p. 534, 1926). Further, in dealing with injection experiments : all these are artificial. It is a contradiction in terms to speak of injecting fluid into the eye under "normal pressures." In addition, I think it is difficult to admit without reservation that Chinese ink "moves with the fluid" in which it finds ifself "until it is filtered out of it by the membranes through which the flutid passes." On the other hand, apart from the disturbed conditions of pressure which the injection entails, substances even as inert as inks have been demonstrated to excite a considerable cellular reaction in the eye (see Hamburger), and are to some extent, at any rate, actively eliminated by the phagocytic activity of the endothelial cells lining the chambers of the eye, just as all foreign material is eliminated from any part of the body into which it may be introduced. The behaviour of solid particles of Chinese ink in this case is merely a demonstration of how foreign substances are dealt with when they are introduced artificially into the eye, and can carry no legitimate conclusion as to the movement or stagnation of the intra-ocular fluids under normal circumstances. Ulbrich's observation is the most perfect demonstration of a pressure circulation that has been recorded in the literature (p. 89). Finally, the clinical evidence of glaucoma certainly demonstrates the existence of a circulation (cp. p. 106), but gives no clue as to its origin or nature.

If the aqueous humour is formed by a pressure transudation as Leber suggested and circulates actively through the eye, and if it is not a product of thermo-dynamical equilibrium, then the one fact that it contains more negative ions (chlorides, etc.) than the plasma, forces one, to my mind, to postulate that it is formed by a gland actively secreting saline (p. 103), and secreting (inexplicably) just the amount required by a dialysate. I do not think there can be any question as to the accuracy of the chemistry here, for, neglecting my own analyses which invariably showed such an excess very definitely, the excess has been demonstrated by Mestrezat and Magitot, Creveld, Gala, Rados, Ascher, and many others (p. 29). These all used modern methods of considerable accuracy, and the phenomenon was found to occur in all the animals upon which estimations have been made-man, horse, rabbit, dog, sheep, ox, etc. Such an excess cannot be accounted for by a theory of simple transudation. This fact was known, indeed, to Leber, and he himself could not explain it.

Priestley Smith is of the opinion that the conclusion that the aqueous humour is in "osmotic equilibrium" " has been reached on insufficient data, and, as it is supported, is "hardly safe." I can find no evidence, experimental or clinical, in my experience or in my reading, which can withstand reasonable and logical criticism, and which is incompatible with the hypothesis that the intra-ocular fluids are a dialysate upon which is superimposed a circulation. I have examined the alternative theories put forward from time to time and consider that in one place or another they all break down. It is true that the thesis was offered, not even as a theory,

"The term " osmotic equilibrium" does not express the condition of dialysis as well as "thermo-dynamic equilibrium," a state which depends upon the interaction of hydrostatic, electrical, and chemical, as well as osmotic forces. It is to be remembered that, as in all living systems, the equilibrium is a dynamic, and not a static one. 
but only as a working hypothesis without any claim to infallibility or permanency; if substantial evidence can be brought against it, then we hope to have the pleasure of building up another. But if the aqueous humour possesses all the very definite and complicated chemical and physical properties of a dialysate, both in its normal condition and in variations from the normal, if it can be shown, not to be in "supposed osmotic equilibrium," but to fulfil, in experimental fact, the complex and peculiarly exacting hydrostatic, osmotic, electrical, and chemical conditions required by a system in thermo-dynamical equilibrium with capillary plasma, and if at the same time no adequate objection is put forward or alternative and unassailable explanation is advanced, there seems to be no valid reason at the present time against the adoption of such a working hypothesis.

\section{ANNOTATIONS}

\section{The International Congress of Ophthalmology, 1929}

We have much pleasure in announcing that the re-establishment of International Congresses of Ophthalmology is an accomplished fact. The next Congress will take place in Holland in September, 1929. This satisfactory conclusion to negotiations which have spread over several years is due in the first place to the perseverance and tact of the Anglo-American Committee which was appointed by the English-Speaking Conference of Ophthalmology. Of the members of that Committee the chief burden has fallen upon $\mathrm{Mr}$. Treacher Collins, the chairman, and Mr. Leslie Paton, the secretary. Even their efforts, however, might have been frustrated had it not been for the genius and geniality of Dr. van der Hoeve, of Leyden, who presided over the Conference of delegates from all nations which met in July at Scheveningen. The congratulations and thanks of all ophthalmologists throughout the world are due, and will be willingly and gratefully accorded, to these three intermediaries in the re-establishment of plenary international relations so far as they affect ophthalmology.

\section{William Mackenzie Memorial}

On another page of this number will be found an announcement of a proposed memorial to the great Scottish ophthalmologist of the last century. Details will be found in the note; and it is only 